\title{
Prevention and control of intensive care unit-acquired carbapenem-resistant Klebsiella pneumoniae: need for a multimodal approach
}

\author{
Arta Karruli ${ }^{1}$, Roberto Andini ${ }^{1}$, Antonio Corcione ${ }^{2}$, Emanuele Durante-Mangoni ${ }^{1}$ \\ ${ }^{1}$ Department of Precision Medicine, University of Campania 'L. Vanvitelli', and Unit of Infectious \& Transplant Medicine, AORN Ospedali dei \\ Colli-Ospedale Monaldi, Naples, Italy; ${ }^{2}$ Department of Critical Care, AORN Ospedali dei Colli-Ospedale Monaldi, Naples, Italy \\ Correspondence to: Emanuele Durante-Mangoni, MD, PhD. Ospedale Monaldi, Piazzale E. Ruggieri snc, 80131 Napoli, Italy. \\ Email: emanuele.durante@unicampania.it. \\ Provenance: This is an invited article commissioned by the Section Editor Dr. Guo-wei Tu (Department of Critical Care Medicine, Zhongshan \\ Hospital, Fudan University, Shanghai, China). \\ Comment on: Li M, Wang X, Wang J, et al. Infection-prevention and control interventions to reduce colonisation and infection of intensive care \\ unit-acquired carbapenem-resistant Klebsiella pneumoniae: a 4-year quasi-experimental before-and-after study. Antimicrob Resist Infect Control \\ 2019;8:8.
}

Submitted Sep 05, 2019. Accepted for publication Sep 26, 2019.

doi: 10.21037/atm.2019.09.130

View this article at: http://dx.doi.org/10.21037/atm.2019.09.130

There is a significant increase in the occurrence of carbapenem-resistant Klebsiella pneumoniae (CRKP) blood-stream infections among identified carriers of CRKP, especially in critically ill patients dwelling in intensive care units (ICU). This is emerging as a particularly critical problem due to increasing resistance and mortality among ICU patients in developed as well as developing countries (1).

Until now, antibiotics were regarded as the main antiinfective strategy for patients with multi-drug resistant (MDR) bacterial infections in ICU. Modern antibiotic stewardship and infection prevention and control (IPC) have not fully succeeded in the prevention of antibiotic resistance and mortality caused by MDR organisms. There is an emerging need for newer strategies incorporating different approaches against bacterial resistance (2).

We read with interest the recent study by $\mathrm{Li}$ and colleagues (3), who used several interventions to try curtailing the epidemic spread of CRKP in a general ICU ward in China, along 4 sequential stages:

(I) Baseline period, when no specific intervention was implemented;

(II) Period 1, divided in 3 levels of intervention intensity based on risk factors for MDR, and proven MDR colonization/infection;

(i) interventions used were: (ii) non level dependent: active surveillance cultures, de-escalation therapy;

(iii) level dependent: precautions of contact, isolation of patients with MDR, cohorting of patients and medical care, disinfection and sterilization;

(iv) targeted bundles, based on the site of infection.

(III) Period 2 (IPC-modified interventions), which in addition to all period 1 interventions had also entailed: medical staff education, contact precautions of shared equipment, terminal room disinfection;

(IV) Period 3, during which follow-up was completed.

All these complex interventions translated into an important and statistically significant reduction of ICUacquired CRKP colonization/infection, including bloodstream infection due to catheters, ventilator associated pneumonia, infections of skin and soft tissues. During all periods, no cases of catheter-associated urinary tract infection were detected.

In this experience, active surveillance cultures were carried out immediately and as a routine during the hospital stay in the ICU ward. Authors used various sampling sites/samples, including nasopharynx (swabs), sputum, endotracheal aspirate, urinary tract, and other possible 


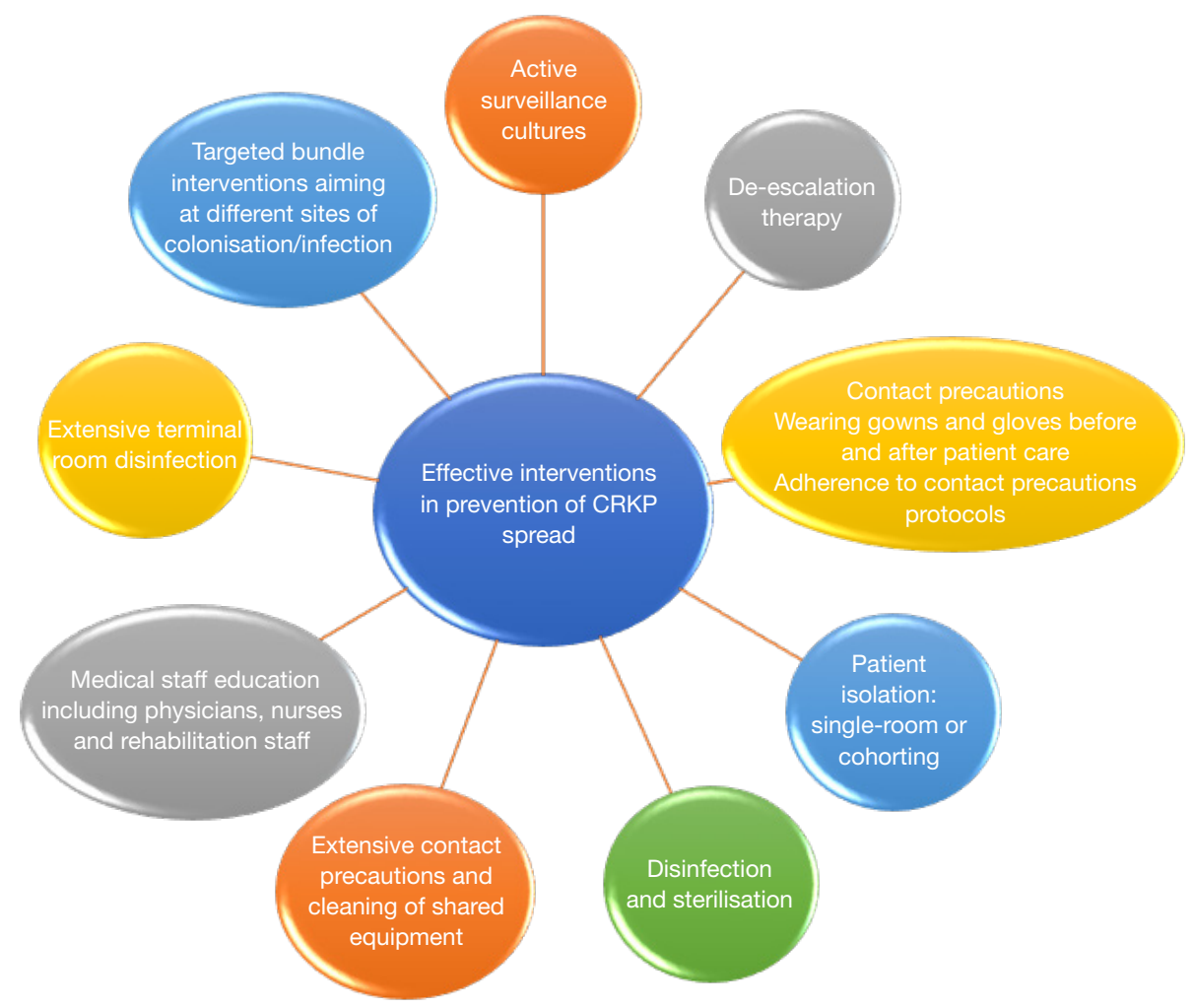

Figure 1 Effective interventions in prevention of CRKP spread. CRKP, carbapenem-resistant Klebsiella pneumoniae.

infection sites. However, they did not declare whether rectal swabs for CRKP were performed. Lack of rectal swab testing for CRKP would likely underestimate the real number of patients colonized by CRKP, a common host of the gastrointestinal tract (4).

IPC effectiveness has a major impact on reducing antibiotic resistance spread and related mortality. By preventing infection, IPC also aids in reducing antibiotic use, further limiting antibiotic resistance. Noteworthy, in the study by $\mathrm{Li}$ et al. (3), among the various interventions used to prevent colonization/infection there was antimicrobial de-escalation therapy. However, the individual impact of each intervention was not analysed, leaving unmeasured the impact of de-escalation. Moreover, whether any new antibiotic was used compared to the baseline period or colonized subjects underwent any decolonization treatment remains undefined (3).

Preventing colonisation/infection of CRKP in ICU patients is one of the main goals of modern medicine. It is far more complex than just preventing colonisation/ infection. Rather, it is the result of multimodal interventions with various purposes (Figure 1): the active search of colonized patients; the prevention of colonisation among other patients admitted in the same ICU ward; the prevention of infection among already colonized patients with MDR; and the reduction of mortality among infected patients. Therefore, for IPC to be effective, all these phases must be implemented (5). A more detailed description of these interventions is lacking in the study by $\mathrm{Li}$ et al. Also missing was a more detailed description of the rate of ICU admissions protected from colonization, the rate of colonized patients who did not proceed to infection and, in the infection group, the mortality rate during the four periods.

In the follow-up period of this study, a decline in the number of ICU acquired colonization/infection was observed, despite of a considerable number of colonized/ infected patients on admission. Thus, preventing colonization/infection of ICU patients in a ward where exposure from other patients is major can be viewed as an achievement of a great importance. Even though this was not a case control study and the number of patients was limited, there is lack of information regarding risk factors, antibiotic use, and prior hospital stay in patients admitted to 
the ICU.

Several studies have addressed the topic of the effectiveness of various interventions in colonization/ infection prevention in ICU wards in developed countries (6-9). In contrast, data are limited for developing countries. The study by $\mathrm{Li}$ et al. is one of the first performed in an emerging country. The interventions used in this study could have a limited feasibility in other developing countries, as they require additional costs, implying hospital facilities structured with isolation rooms. Also, many large hospitals are overcrowded and understaffed and suffer from technological gaps. Finally, staff adherence to the new interventions may not be optimal.

We remain in a never-ending fight against fast evolving MDR microorganisms. In the pre-antibiotic era, pathogenic bacteria were often prevailing. After antibiotics discovery, they endured in silence an apparent defeat to re-emerge as winners by becoming resistant. It is now time for us to react, although the response is far more complex than just another new antibiotic to discover. Indeed, bacteria will keep developing resistance to newer antibiotics, leaving us with limited weapons. The only alternative remaining will be preventing them from causing harm to our patients.

\section{Acknowledgments}

Funding: E Durante-Mangoni and R Andini were supported by an NIH grant no. HHSN272201000039C, subcontract 3004788744.

\section{Footnote}

Conflicts of Interest: E Durante-Mangoni received grant support and speaker's honoraria from Pfizer, MSD, Angelini, Bio-Merieux, Abbvie, Sanofi-Aventis, Medtronic, and DiaSorin. The other authors have no conflicts of interest to declare.

Ethical Statement: The authors are accountable for all aspects of the work in ensuring that questions related to the accuracy or integrity of any part of the work are appropriately investigated and resolved.

\section{References}

1. Tian L, Tan R, Chen Y, et al. Epidemiology of Klebsiella pneumoniae bloodstream infections in a teaching hospital: factors related to the carbapenem resistance and patient mortality. Antimicrob Resist Infect Control 2016;5:48.

2. Bassetti M, Poulakou G, Ruppe E, et al. Antimicrobial resistance in the next 30 years, humankind, bugs and drugs: a visionary approach. Intensive Care Med 2017;43:1464-75.

3. Li M, Wang X, Wang J, et al. Infection-prevention and control interventions to reduce colonisation and infection of intensive care unit-acquired carbapenem-resistant Klebsiella pneumoniae: a 4-year quasi-experimental before-and-after study. Antimicrob Resist Infect Control 2019;8:8.

4. Borer A, Eskira S, Nativ R, et al. A multifaceted intervention strategy for eradication of a hospital-wide outbreak caused by carbapenem-resistant Klebsiella pneumoniae in Southern Israel. Infect Control Hosp Epidemiol 2011;32:1158-65.

5. Durante-Mangoni E, Andini R, Zampino R. Management of carbapenem-resistant Enterobacteriaceae infections. Clin Microbiol Infect 2019;25:943-50.

6. Ben-David D, Masarwa S, Fallach N, et al. Success of a National Intervention in Controlling Carbapenemresistant Enterobacteriaceae in Israel's Long-term Care Facilities. Clin Infect Dis 2019;68:964-71.

7. Karampatakis T, Tsergouli K, Iosifidis E, et al. Impact of active surveillance and infection control measures on carbapenem-resistant Gram-negative bacterial colonization and infections in intensive care. J Hosp Infect 2018;99:396-404.

8. Mularoni A, Martucci G, Douradinha B, et al. Epidemiology and successful containment of a carbapenem-resistant Enterobacteriaceae outbreak in a Southern Italian Transplant Institute. Transpl Infect Dis 2019;21:e13119.

9. Bianco A, Quirino A, Giordano M, et al. Control of carbapenem-resistant Acinetobacter baumannii outbreak in an intensive care unit of a teaching hospital in Southern Italy. BMC Infect Dis 2016;16:747.

Cite this article as: Karruli A, Andini R, Corcione A, DuranteMangoni E. Prevention and control of intensive care unitacquired carbapenem-resistant Klebsiella pneumoniae: need for a multimodal approach. Ann Transl Med 2019;7(Suppl 8):S325. doi: $10.21037 / \mathrm{atm} .2019 .09 .130$ 